\title{
Ontología Orientada a Objetos: UNA NUEVA TEORÍA DE TODO
}

\author{
Object-Oriented Ontology: A NeW Theory of EVERything \\ Luis Alberto Jiménez Morales \\ DOI: 10.26754/ojs_arif/a.rif.202014044
}

Graham Harman (2018). Object-Oriented Ontology: A New Theory of Everything. Great Britain: Penguin Books. 295 páginas.

La Ontología Orientada a Objetos (de ahora en adelante triple OOO) es relativamente una nueva corriente dentro de los problemas contemporáneos de la filosofía. Uno de sus principales exponentes es el filósofo norteamericano Graham Harman. Para comprender la filosofía de Harman, es necesario determinar cuál es el problema fundamental que le preocupa. Su propuesta se articula como un diálogo crítico con las ciencias naturales y con las implicaciones de la filosofía moderna y contemporánea.

Así pues, el objetivo de esta reseña es uno de sus más recientes trabajos: ObjectOriented Ontology: A New Theory of Everything, que apareció en Penguin Books en el 2018. Hablamos de un texto que denota un riguroso y loable esfuerzo por hacer una introducción de la OOO a un amplio público. Este ensayo muestra una provocadora e incisiva crítica a las tradiciones filosóficas que consideran que la realidad es algo construido por el lenguaje, las relaciones de poder o las prácticas culturales. Por el contrario, la OOO sostiene que el mundo externo existe independientemente de la conciencia y percepción humana. Los objetos se presentan con independencia de la conciencia del sujeto. Los objetos no necesitan de la conciencia del sujeto para existir, esto demuestra una extensiva crítica a los principios de la filosofía moderna de René Descartes e Immanuel Kant. No obstante, Harman piensa que hay una brecha entre el conocimiento y la realidad, existe una imposibilidad de acceder directamente a los objetos. Harman piensa la relación con los objetos desde el noúmeno kantiano, es decir, hay una imposibilidad de conocer las cosas como son en realidad. 
El primer capítulo llamado Una nueva teoría de todo comienza a articular una discusión sobre el papel de las ciencias naturales en la interpretación de la realidad. Ciertamente, la física a través de la teoría de cuerdas ha intentado erigirse como una teoría de todo. Probablemente, la teoría de cuerdas es aquella que podría explicar con mayor éxito la composición de la materia y la estructura del cosmos (Harman, 2016). Sin embargo, Harman considera que la teoría de cuerdas es incapaz de fundamentar una teoría de todo, puesto que se precipita en cuatro errores fundamentales: 1) cualquier cosa que exista necesita ser física. Esta primera aseveración se encuentra en el fisicalismo que sostiene que las entidades no materiales carecen de interés. En su ensayo intitulado Immaterialism Harman proporciona un ejemplo que refuta esta cuestión, la Compañía Neerlandesa de las Indias Orientales (VOC) muestra que algunos de sus elementos materiales constantemente desaparecen y surgen otros, pero eso no implica que la VOC desaparezca. "La Compañía Neerlandesa de las Indias Orientales no era solamente un conjunto de átomos y cuerdas, sino una larga extensión capaz de sobrevivir al movimiento y a la desaparición de pequeños elementos" (Harman, 2018: 29). La VOC no es solamente la suma de sus elementos materiales. 2) Cualquier cosa que exista debe ser básica y simple. Esta segunda aseveración se encuentra en el pequeñismo, que sostiene que las cosas reales en cualquier situación tienen diminutos elementos de los que están compuestas. No obstante, Harman considera que el pequeñismo ignora el fenómeno de la emergencia. La emergencia se demuestra cuando los elementos se juntan para formar uno nuevo. Veamos la situación: cuando dos personas contraen matrimonio, surge una entidad nueva e impredecible. 3) Cualquier cosa que existe debe de ser real. Esta afirmación se encuentra expresada en el anti-ficcionalismo, la física y en especial la teoría de cuerdas está orientada al descubrimiento de entidades físicas reales, las ficticias carecen de interés. Uno de los aspectos centrales de la OOO es defender que una teoría de todo debe de ser capaz de dar cuenta de entidades no físicas en la misma medida en que se ocupa de las entidades físicas. 4) Cualquier cosa que exista debe de ser capaz de ser exactamente expresada un proposicional literal. La falsedad de la cuarta aseveración se encuentra en el literalismo, cuya crítica está precisamente vinculada con el desarrollo del segundo capítulo.

En el segundo capítulo Estética es la raíz de toda la filosofía Harman intenta explicar uno de los principales postulados de la OOO, que considera que el acceso a la realidad no puede darse de forma directa, ya que la realidad está colmada de un fondo impenetrable que se asemeja al noúmeno kantiano. Entonces, si el acceso a la realidad es imposible de forma directa, solo queda intentar acceder a la realidad de modo indirecto. El autor sostiene que el arte es una forma de acceder a la 
realidad de forma indirecta. Asimismo, un poco conocido ensayo de 1914 de José Ortega y Gasset llamado Phenomenology and Esthetics, donde existe una reflexión sobre la metáfora, ha proporcionado a la OOO el material para abordar esa situación. En primer lugar, la metáfora no intenta darnos pensamientos o percepciones del objeto, ya que nos da una visión externa del objeto, la metáfora nos devuelve a la cosa como es en realidad, una cosa en sí. En segunda instancia, la metáfora es asimétrica, por ejemplo: el poema de López Pico dice que "el ciprés es como el espectro de una llama muerta", podríamos decir que ninguno de los dos objetos se encuentran en el mismo plano, ya que el primero asume el rol de objeto y el segundo dibuja las cualidades de aquel objeto. La OOO sostiene que el objeto real se retrae del acceso directo, por lo tanto en el caso del poema, el ciprés se retrae y lo que queda son las cualidades dibujadas en la llama. Según Harman, lo que sucede en la metáfora es que el acceso al ciprés no es representado de manera directa, sino que la metáfora devuelve una amalgama de realidad formada desde el lector, es decir, a partir del ciprés-objeto y la flama-cualidades, la metáfora nos arroja un nuevo objeto, un ciprés-llama. El arte acopla activamente al espectador y al objeto estético, es lo que Harman llama teatricalidad, el espectador sustituye al objeto. El arte no tiene que ver con una producción de conocimiento stricto sensu, sino con la creación de nuevas cosas en sí mismas (Harman, 2018). El arte muestra a la filosofía que el acceso a la realidad es por medio de una relación indirecta.

En el tercer capítulo Sociedad y política Harman establece un diálogo crítico con la Actor-Network Theory (ANT) de Bruno Latour, cuyos fundamentos plantean una ontología plana que intenta darle el mismo peso a entidades humanas y no humanas, las cuales entran en una interacción simétrica. "La ANT [...], reduce a los actores a efectos mutuos unos sobre otros" (Harman, 2018: 107). La ANT plantea que existe un acceso directo a los objetos, reduciendo los objetos a lo que pueden hacer. Por ejemplo, si pensamos la Revolución pasteuriana desde el enfoque de la ANT, ésta nos diría que no solamente se debe de poner atención al genio que fue Louis Pasteur, sino también a los entes no-humanos que jugaron un papel importante en este evento. La ANT sostiene que las relaciones sociales son simétricas, la realidad está constituida por relaciones reciprocas. Por el contrario, dice Harman: "Lo que interesa a la OOO, en cambio, es el modo en que mi encuentro con una llama o un llamado ético forma un nuevo objeto en su propia verdad, y no solo una interacción externa entre dos entidades permanentemente separadas" (Harman, 2018: 107).

Con relación a la política Harman considera que la OOO tiene una coincidencia muy estrecha con la perspectiva de Latour, puesto que en un reciente trabajo 
llamado An Enquiry Into Modes of Existence sostiene en un tono casi hobbesiano, que el conocimiento político no es obtenible y ni siquiera deseable, pues la política surge en cualquier lugar donde exista un problema, tomando la forma de una disputa entre sujetos interesados en esa cuestión, dicha disputa solo finaliza con una decisión, lo cual excluye al conocimiento. $\mathrm{Al}$ igual que la ANT, la OOO sostiene que la política tampoco es el sitio donde se alcanza el conocimiento o la verdad.

En el cuarto capítulo llamado Relaciones indirectas Harman regresa a la discusión que quedó pendiente en el capítulo tres. Comienza reconociendo que los objetos reales se resisten a ser sujetados por las teorías humanas, percepciones o acciones prácticas, el modo de relación con los objetos debe darse de forma indirecta, tal como sucede en la metáfora. En este sentido, Harman considera que los objetos tienen una estructura cuádruple (Objeto Real, Cualidades Sensuales, Cualidades Reales y Objeto Sensual), lo que se pretende con esta estructura es distinguir dos tipos de objetos y dos tipos de cualidades. La OOO sostiene que el conocimiento científico es literal, por ello no puede acceder al objeto puesto que se retrae, en todo caso el conocimiento debe intentar acceder al Objeto Sensual para arribar a las Cualidades Reales. Harman sostiene: "Hemos dicho también que el conocimiento no puede ser metafórico sino literal, $[\ldots]$ en el conocimiento el objeto está ante nosotros directamente, pero está construido de un manojo de misterios" (Harman, 2018: 182). Por ello, en el pensamiento científico el objeto se retrae.

En el quinto capítulo Ontología Orientada a Objetos y sus Rivales Harman intenta demostrar que la OOO tiene una diferencia significativa con relación a las propuestas teóricas de dos filosófico continentales muy renombrados, Jacques Derrida y Michel Foucault. Harman sostiene que una de las principales propuestas de la filosofía de Derrida es formular una crítica a la presencia. De hecho, el filósofo francés señala que es la principal equivocación de la tradición intelectual de Occidente. Derrida considera que es imposible pretender que el significado de una palabra o un texto sean aislados de la posibilidad de numerosos contextos en los que pueden ser interpretados, las palabras están inmersas en una amplia red de significados. Para Harman, Derrida asume una posición holista anti-realista que considera que todo el leguaje es metafórico, por el contrario la OOO es una teoría realista que asume una distinción entre el lenguaje literal y metafórico. En un sentido análogo, Michel Foucault muestra preocupación en el concepto de unidad, es decir, lo que llamamos unidad no es más que un conjunto de prácticas discursivas que le dan sentido y unidad. Harman considera que en la filosofía foucaultiana la agencia de los objetos se disuelve en las prácticas discursivas, ciertamente los objetos encarnan esas prácticas pero no tienen realidad. 
Finalmente, el último capítulo Varios enfoques para la Ontología Orientada a Objetos intenta destacar las concordancias del trabajo de varios teóricos que están inmersos en la OOO. Timothy Morton es uno de los principales representantes de la OOO, rechaza la idea moderna de que el hombre toma distancia del mundo o que está por encima de éste, el hombre se encuentra en el mundo al igual que los hiperobjetos para acuñar el término de Morton. Harman incita pensar la realidad desde una ontología plana. En suma, podríamos decir que el ensayo Object-Oriented Ontology: A New Theory of Everything es un riguroso esfuerzo por hacer que esta nueva escuela de pensamiento sea comprendida por un amplio público. Una escuela que está preocupada por la agencia de los objetos, sin reducirlos únicamente a sus cualidades.

$$
\begin{array}{r}
\text { Luis Alberto Jiménez Morales } \\
\text { Univ.Autónoma de la Ciudad de México } \\
\text { jmluisalberto@gmail.com }
\end{array}
$$

\section{BIBLIOGRAFÍA}

Harman, G. (2016): Immaterialism: Objects and Social Theory. United States: Polity Press.

Harman, G. (2018): Object-Oriented Ontology: A New Theory of Everything. Great Britain: Penguin Books.

Latour, B. (2018): An Inquiry into Modes of Existence. United States: Harvard University Press.

Morton, T. (2013): Hyperobjects: Philosophy and Ecology after the End of the World. United States: University of Minnesota Press. 